\title{
Coherent backscattering and localization in a self-attracting random walk model
}

\author{
R. Lenke ${ }^{1,2}$, R. Tweer ${ }^{1}$, and G. Maret ${ }^{1, a}$ \\ 1 University of Konstanz, Box 5560, 78457 Konstanz, Germany \\ 2 Institut Charles Sadron, 6 rue Boussingault, 67083 Strasbourg Cedex, France
}

\begin{abstract}
Intensity propagation of waves in dilute 2D and 3D disordered systems is well described by a random walk path-model. In strongly scattering media, however, this model is not quite correct because of interference effects like coherent backscattering. In this letter, coherent backscattering is taken into account by a modified, self-attracting random walk. Straightforward simulations of this model essentially reproduce the results of current theories on "non-classical" transport behavior, i.e. Anderson localization in $1 \mathrm{D}$ and 2D for any amount of disorder and a phase transition from weak to strong localization in 3D. However, in the strongly scattering regime corrections are necessary to account for the finite number of light modes due to their non-vanishing lateral extention. Within our model this correction leads to the observation that strong localization does not take place.
\end{abstract}

PACS. 42.25.Dd Wave propagation in random media - 71.23.An Theories and models; localized states

\section{Introduction}

The problem of light propagation in 2D and 3D disordered systems can be treated by solving Maxwell's equations, in principle [1]. However, this is a very complicated task - analytically as well as numerically - and considerable simplifications are necessary, normally, in order to make qualitative and quantitative predictions. The most common approach uses a Green's function formalism $[2,3]$ resulting in a diagrammatic path model of amplitudes and - under the assumption that there are no correlations - in a path model of intensities. The latter can be mapped onto a random walk of photons with a characteristic Boltzmann diffusion constant $D_{\mathrm{B}}=v_{\mathrm{E}} \ell^{\star} / d, v_{\mathrm{E}}$ being the energy transport velocity, $\ell^{\star}$ the transport mean free path of uncorrelated, random steps and $d$ is the dimensionality.

Most experimental results are accounted for by the diffusion model, even in cases where the diffusion constant cannot be forecast explicitely ${ }^{1}$. One result of the diffusion theory is that the total transmitted intensity through a non-absorbing 2D or 3D slab of thickness $L$ follows Ohm's law $\left(\right.$ i.e. $\left.\propto L^{-1}\right)$. Through a $1 \mathrm{D}$ randomly layered stack of thickness $L$, however, the average transmitted inten-

\footnotetext{
a e-mail: georg.maret@uni-konstanz.de

1 Extensions of the diffusion model with respect to nonisotropic scattering (i.e. a scattering mean free path $\ell$ of Lambert-Beer's law different from $\ell^{\star}$ ), inter-particle correlations (structure factor) and an effective medium approach are treated in $[2,3]$.
}

sity is proportional to $\exp (-L / \xi)$. This exponential decay corresponds to a behavior which is generally called localization; $\xi$ being the localization length. This exponential law cannot be explained by the above random walk model. Deviations from the random walk model should also appear in $2 \mathrm{D}$ and $3 \mathrm{D}$, especially at high disorder due to the correlation between each path and its reversed counterpart. Both paths have exactly the same length and thus are always in phase at each starting and end point of a loop. This correlation results in a constructive interference enhancement of a factor of two at these points ${ }^{2}$. This so-called coherent backscattering (CB) doubles the probability of light paths to form closed loops in comparison with non-interfering models. As a consequence, $\mathrm{CB}$ reduces the diffusion constant and makes the diffusion "non-classical". In very strongly scattering samples, where $k_{\mathrm{e}} \ell^{\star} \lesssim 1$ (Ioffe-Regel criterion, "e" for effective medium), a "non-classical" transport behavior was predicted, the so-called strong or Anderson localization [4,5].

A twofold intensity enhancement with respect to the incoherent background is also observed outside the sample. It is known as the $C B$-cone [6]. Experimentally, the CB-cone was observed for light and acoustic waves. Nowadays, it represents a powerful tool for the characterization of turbid samples. A CB-induced reduction of the diffusion constant in case of quantum waves was verified for electrons [7] by applying a magnetic field which

\footnotetext{
${ }^{2}$ In case of scalar waves. For vector waves it is approximately a factor of 1.5 on average.
} 
breaks symmetry between the direct and reversed paths. Similarly, optical CB is suppressed by magneto-optical Faraday rotation [8-11] or by reciprocity breaking effects at atomic level [12]. Anderson localization of light in 2D or $3 \mathrm{D}$, solely due to disorder, has not yet been observed beyond any doubt [13-15].

Current theories treating the influence of CB on localization make approximations which are strictly valid only for the weak scattering limit. Predictions for the strong scattering regime, i.e. where $\ell^{\star}$ is of the order of the wavelength, rely on extrapolation. The self-attracting random walk model we present in Section 2 shows all essential features predicted by these theories. This not only suggests its significance but also provides an intuitive physical picture of the influence of $\mathrm{CB}$ on multiple light scattering in strongly disordered media. In addition, it allows to easily account for the non-vanishing lateral extention of light modes as described in Section 3. For simplicity, we only consider the cases of scalar waves and isotropic scattering, i.e. $\ell^{\star}=\ell$.

\section{A self-attracting random walk model}

CB increases the return probability to a former point of the random walk independently of the path length. Consequently it cannot be cast in terms of a modified structure factor and mapped on a renormalized random walk of non-correlated scattering events. The phenomenon of CB therefore results in a deviation from the simple random walk model. Current theories treat $\mathrm{CB}$ in the following way [3]: Due to CB the scattering becomes non-isotropic thus changing $D_{\mathrm{B}}$. However, the non-isotropy depends on $D_{\mathrm{B}}$ itself what necessitates a self-consistent ansatz. Our concept rather follows the (ensemble averaged) evolution of a wavefront in real space and time. Thereto it is necessary to understand the influences of $\mathrm{CB}$ on the wavefront. Let us first consider the CB-cone outside a sample. After averaging out the speckle pattern the cone appears in exact backscattering of an incident plane wave and has a full width at half maximum of about $1 / k_{0} \ell^{\star}$, where $k_{0}$ is the wavenumber in air. When using a pointlike source at a distance $F$ from the sample, CB appears around the source within a region of about $F / k_{0} \ell^{\star}[6]$. The general assumption is now that each scatterer inside a multiple scattering medium can be regarded as a point-like source. Approximating $F$ by $\ell^{\star}$ inside the multiple scattering medium one obtains a "CB-region" with a diameter of about $k_{\mathrm{e}}^{-1}=\lambda_{\mathrm{e}} / 2 \pi$ which is approximately the width of a light mode ( $\lambda_{\mathrm{e}}$ is the effective medium wave length). Of course, this cone is only obtained after averaging over many loops and configurations of the scatterers. In the following, we approximate those CB-regions by Gaussians with a full width of $1 / k_{\mathrm{e}}$. At each scattering event a wavefront is "emitted" which decreases exponentially due to subsequent scattering events. In a random walk without $\mathrm{CB}$, the next scattering event would be chosen at random angle and at a distance $\mathrm{d} r$ with an exponential distribution $\exp \left(-\mathrm{d} r / \ell^{\star}\right)$ according to Lambert-Beer's law. With
CB, however, the wave "feels" already visited points due to the part of the wave that has propagated the reversed path. These points, i.e. the CB-regions, must be taken into consideration by an increased return probability. This situation is comparable to the double slit experiment where the correct scattering pattern can be obtained by a coherent summation of all amplitudes or, as we do because the intensity distribution is known, by preweighting the scattering direction according to the interference pattern. Thus, the probability distribution $p$ in a random walk with CB for the next step to the, say $(n+1)$ th point, is proportional to:

$$
\begin{array}{r}
p\left(r_{n+1}\right)=\mathrm{e}^{-\mathrm{d} r / \ell^{\star}}\left(1+\sum_{i=1}^{n-2} \exp \left[-\frac{\left|\boldsymbol{r}_{n+1}-\boldsymbol{r}_{i}\right|^{2}}{2 \sigma^{2}}\right]\right) \\
\sigma=1 / 2 k_{\mathrm{e}} \\
\mathrm{d} r=\left|\boldsymbol{r}_{n+1}-\boldsymbol{r}_{n}\right|
\end{array}
$$

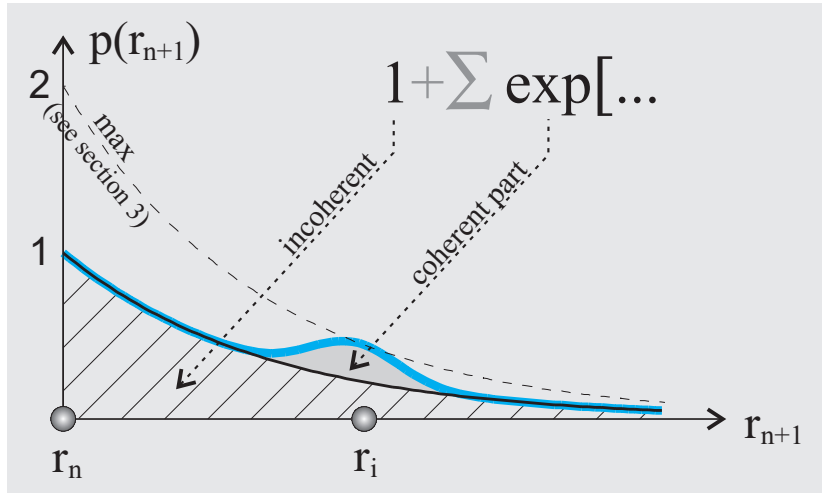

where the $\boldsymbol{r}_{i}$ are the positions of the random walk. The leading exponential factor in equation (1) reflects the step length distribution according to Lambert-Beer's law. The 1 in the sum represents the incoherent summation of all light paths (corresponding to the incoherent background of the CB-cone), the Gaussians stand for the coherent backscattering enhancement. This model corresponds to a self-attracting random walk, the strength of the self-attraction essentially depending on the quantity $1 / k_{\mathrm{e}} \ell^{\star}[16]$. Note that the $(n-1)$ th point is excluded as single scattering does not contribute to CB.

We have studied this self-attracting random walk model by Monte-Carlo simulations. The procedure is based on a "standard" random walk algorithm but with a modified step length distribution according to equation (1). It is worth noting that the random walk does not take place on a lattice, the positions of the scatterers are not predetermined and random walks of different photons are not correlated. The simulations have been performed on a cluster of 12 PCs and took about 4 months. 


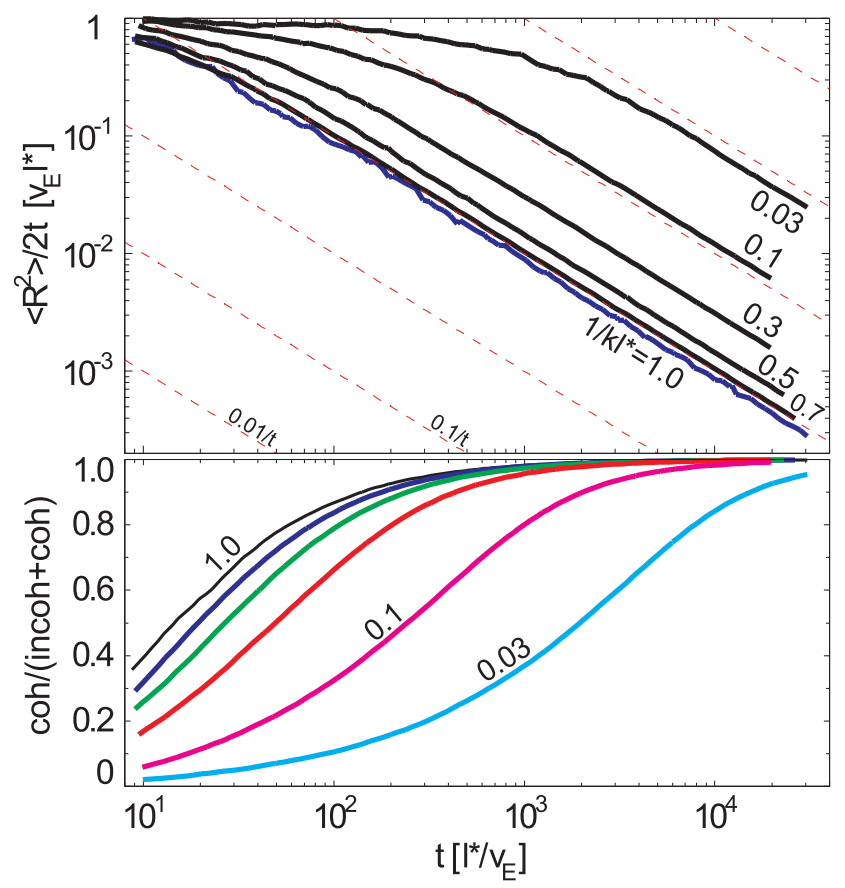

Fig. 1. Time-dependent Monte-Carlo simulations of a 1D selfattracting random walk for different values of $1 / k_{\mathrm{e}} \ell^{\star}$ (scalar waves). Upper graph: Mean square displacement $\left\langle R^{2}\right\rangle$ divided by $2 t$. The dashed lines are guidelines to the eye for strong localization $(\propto 1 / t)$. Lower graph: Return probability due to CB.

The results for the $1 \mathrm{D}$ case are shown in Figure $1^{3}$. The upper part of the figure shows a log-log-plot for different $1 / k_{\mathrm{e}} \ell^{\star}$-values of the mean square displacement $\left\langle R^{2}\right\rangle$, i.e. the average starting-to-end distance of the random walks, divided by $2 t$ as a function of the propagation time $t$. This is the definition of the diffusion constant of a normal random walk in the limit $t \rightarrow \infty$. A value smaller than 1 (in units of $v_{\mathrm{E}} \ell^{\star}$ ) indicates that the diffusion constant is reduced due to the self-attraction. A (locally) non-zero slope $b$ in this plot signifies a diffusive process following another power-law, i.e. $\left\langle R^{2}\right\rangle \propto t^{1+b}[17]$. Photon diffusion is stopped when $b=-1$ and strong localization is reached. Smaller values of $b$ are unphysical in this context. The lower plot of Figure 1 shows the time-dependent probability that a photon is scattered back due to CB to form a closed loop, i.e. the ratio of the coherent part in equation (1) to the incoherent+coherent part. The importance of this plot will be discussed further below. The propagation time $t$ was obtained by multiplying the simulated number of scattering events with the average step length $\langle\mathrm{d} r\rangle$ obtained by the simulations. Due to $\mathrm{CB},\langle\mathrm{d} r\rangle$ itself becomes time-dependent and smaller than $\ell^{\star}$, and also the step length distribution deviates from an exponential one.

\footnotetext{
${ }^{3}$ Note that we added the 1D case only for completeness with respect to other works. As already mentioned in the introduction, wave propagation in $1 \mathrm{D}$ cannot be explained by a simple random walk model of intensities but the amplitudes of all paths must be added up coherently.
}

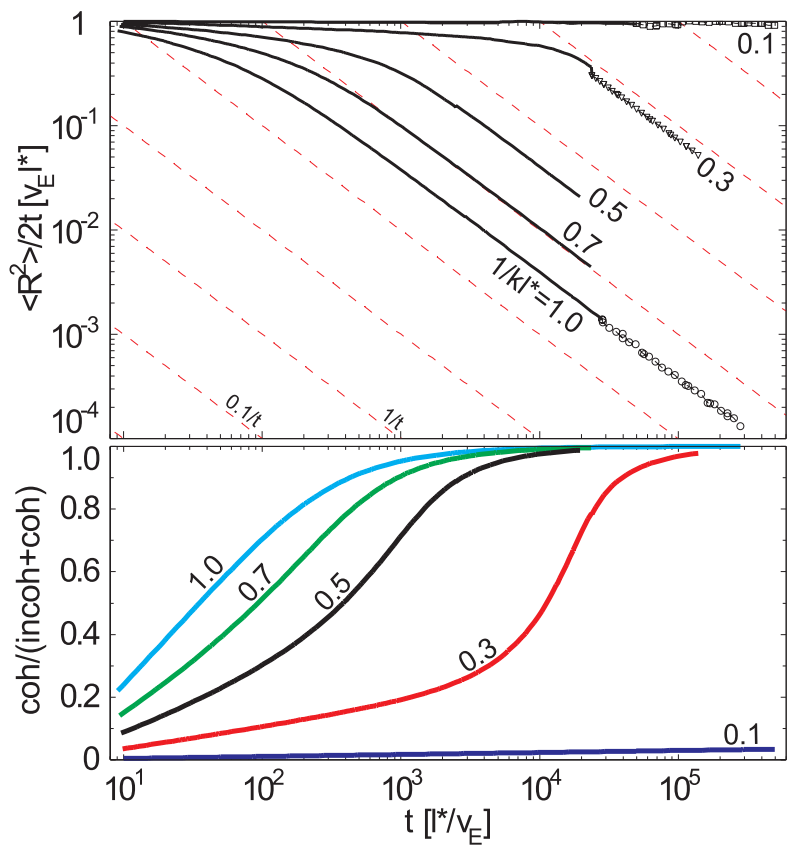

Fig. 2. The $2 \mathrm{D}$ case. Note that for $1 / k_{\mathrm{e}} \ell^{\star}=0.1$ the curve in the lower plot does not saturate but slowly increases. The simulations have been performed in 3 steps for path lengths up to $5 \times 10^{3}, 5 \times 10^{4}$ and $5 \times 10^{5}$ scattering events averaging over about $10^{5}, 10^{4}$ and some hundred photons, respectively. In the graphs, parts of different calculation precision are marked differently.

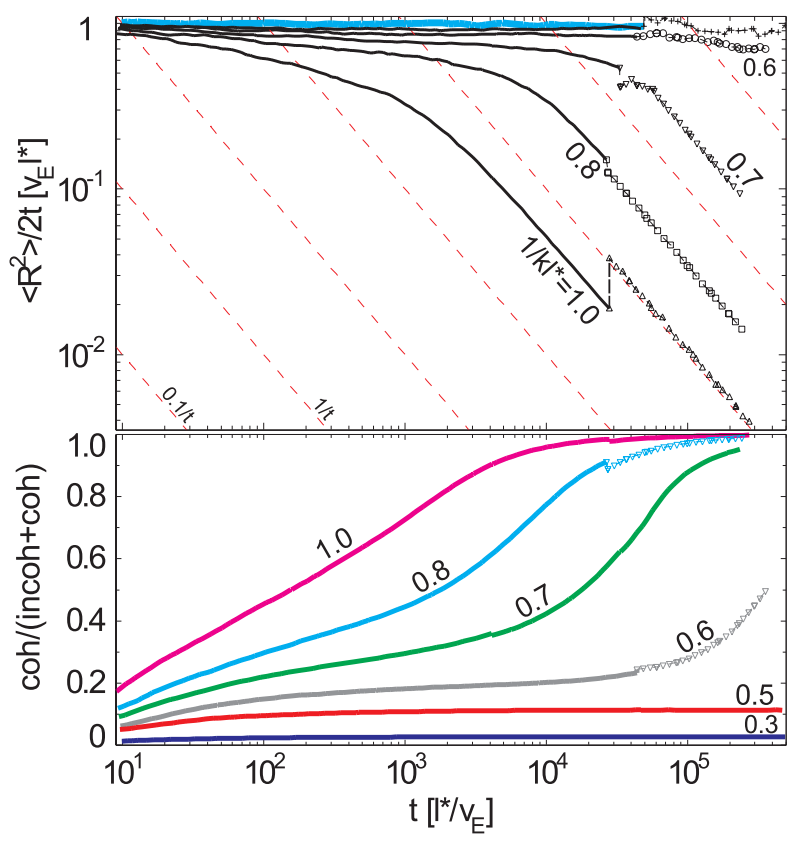

Fig. 3. The $3 \mathrm{D}$ case. In the lower plot, the curves for values $1 / k_{\mathrm{e}} \ell^{\star} \leq 0.5$ tend to saturation. Therefore we estimate $k_{\mathrm{e}} \ell^{\star}=$ 2 for the transition from weak to strong localization. Remark to the jumps in the curves between parts of different precision $(t \approx$ $30000)$ : The simulation error in the slope of the curves is much smaller than the error in their absolute value. Obviously, each photon is localized sooner or later. The center of the localized wave, however, is different for each photon. 


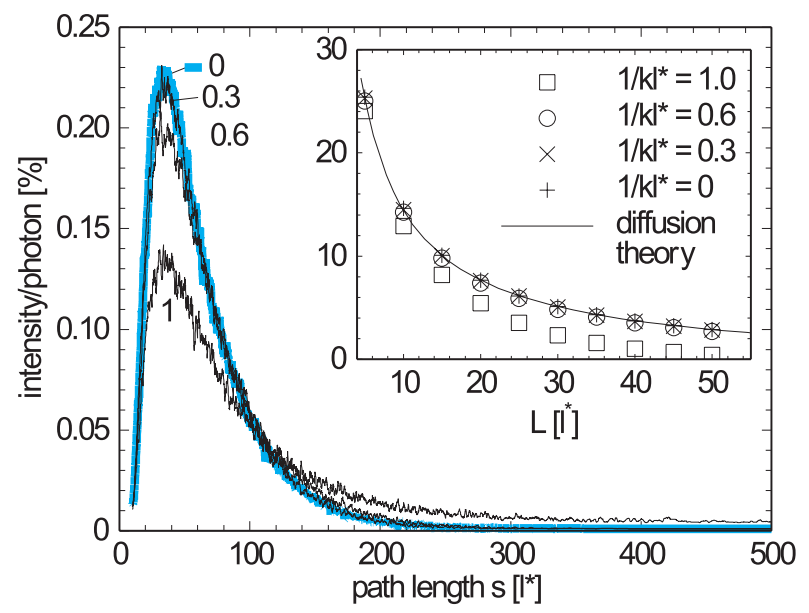

Fig. 4. Monte-Carlo simulations according to the selfattracting random walk model explained in Section 2 of the pulse broadening through a slab of thickness $10 \ell^{\star}$ in 3D for different $1 / k_{\mathrm{e}} \ell^{\star}$-values. Absorption length $\ell_{\mathrm{a}}=10^{4} \ell^{\star}$. Inset: Total transmitted intensity as a function of slab thickness $L$. For $k_{\mathrm{e}} \ell^{\star}=1$ the intensity strongly deviates from diffusion theory.

Figures 2 and 3 show the results for the $2 \mathrm{D}$ and $3 \mathrm{D}$ case. Due to a limited calculation power the behavior of $\left\langle R^{2}\right\rangle / 2 t$ in $3 \mathrm{D}$ is not quite clear for values $1 / k_{\mathrm{e}} \ell^{\star} \approx 0.5$. However, by studying the return probability (lower plots) of the $1 \mathrm{D}$ and $2 \mathrm{D}$ case as well as of the $3 \mathrm{D}$ case for $1 / k_{\mathrm{e}} \ell^{\star}>0.6$, it turns out that the onset of strong localization takes place when the return probability due to CB goes to 1 . This criterion has the advantage that the error bars of the values $c o h /(i n c o h+c o h)$ seem to reduce faster with increasing number of simulated photons than those of the mean square displacement, thus giving a more reliable indication for the onset of localization.

Within the simulated path lengths, our results reproduce the behavior which was found by other theories: strong localization in $1 \mathrm{D}$ and $2 \mathrm{D}$ and a phase transition from weak to strong localization in $3 \mathrm{D}$. We find a critical value $^{4}$ of $k_{\mathrm{e}} \ell^{\star} \approx 2$. The differences in the dimensionalities arise from the fact that the average density of scattering points in a random walk of $n$ steps is proportional to $n^{(1-d / 2)}$ [18]. Our model gives a very clear picture of the influence of $\mathrm{CB}$ on multiple light scattering and it can be studied by Monte-Carlo simulations. For example, Figure 4 shows the simulated pulse broadening and total transmitted intensity of light propagating through a slab, for different values of $1 / k_{\mathrm{e}} \ell^{\star}$.

\section{Corrections due to strong scattering}

As already mentioned in the introduction, current localization theories make approximations which are strictly

\footnotetext{
${ }^{4}$ Of course, this value scales with the precise extention of the CB-region. In another Ansatz, calculating (in the diffusion limit) the CB-cone inside a turbide 2D medium, we obtain a cone which is twice as large.
}

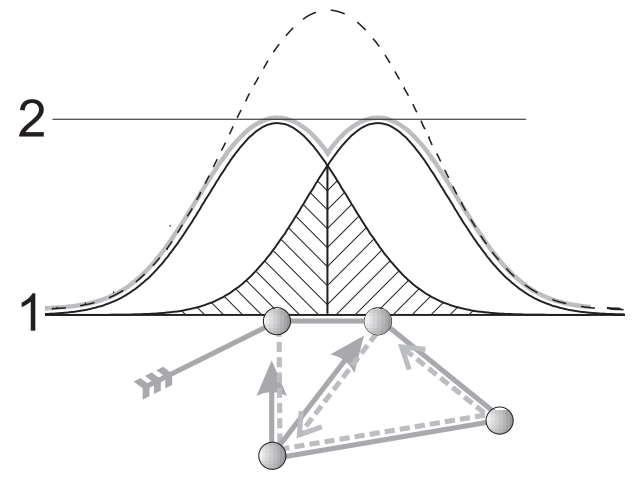

Fig. 5. If two points of a random walk are closer together than $k_{\mathrm{e}}^{-1}$ their CB-regions overlap. Simple summation of both curves results in an enhancement factor larger than 2 . Therefore we have chosen a probability distribution which is given by the envelope (gray curve). Numerically this is carried out as follows: When choosing the next step, we first start as before according to equation (1). The choice of $\mathrm{d} r$ tells us if the next step corresponds to incoherent or to coherent scattering into the CB-region of a certain former scattering event. If however, in case of coherent scattering, this step lies closer to another former scattering event (i.e. if it belongs to the hatched region of the neighboring scattering event), this choice is rejected. Thus, a probability distribution according to the envelope of the Gaussians is obtained.

valid only in the weak scattering limit. This is also the case for the model we have developed so far as we will see in the following. CB creates a twofold interference enhancement at most. Consequently, the dashed line in the drawing to equation (1) indicates the maximum values for the coherent plus incoherent part resulting in a maximum value for the ratio $c o h /($ incoh + coh $)$ of 0.5 . However, in Section 2 we have shown that the onset of localization happens when this ratio approaches 1 which - as we see now - is not possible. "Microscopically", the reason for this contradiction is the following (see Fig. 5): In equation (1) the Gaussian curves have an extention of $k_{\mathrm{e}}^{-1}$. If two points of a random walk are closer than $k_{\mathrm{e}}^{-1}$, the Gaussians overlap resulting in a CB-enhancement larger than 2. Actually, light sources much closer than $k_{\mathrm{e}}^{-1}$ should behave like a single light source in our photon random walk model and should therefore only be counted once (or, if they are counted twice, the incoherent background must be counted twice, too). We accounted for this by replacing the sum of the Gaussians in equation (1) by their envelope (see Fig. 5) in all simulations. This obvious approximation takes the two most important criteria mentioned above into account: No interaction between widely separated scatterers. Two scatterers lying at the same position are counted only once. The results of this refined model are shown in Figures 6 8. Now the relative amount of coherent backscattering is always smaller than 0.5. The diffusion constant is still decreased by coherent backscattering but there no indication of strong localization is seen. In $1 \mathrm{D}$ and $3 \mathrm{D}$ the diffusion constant converges towards a constant finite 


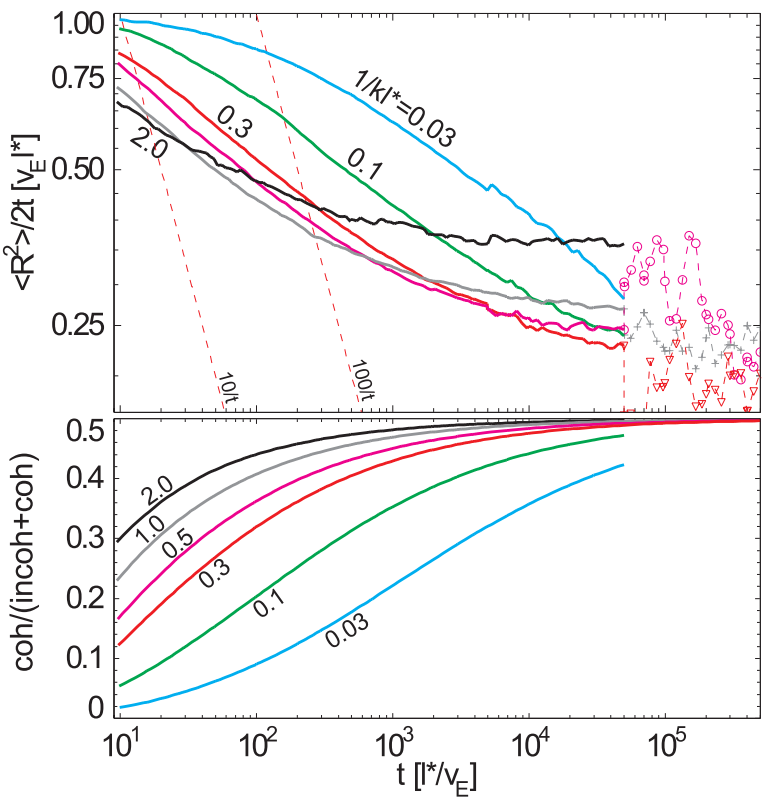

Fig. 6. Time-dependent simulations of a $1 \mathrm{D}$ self-attracting random walk corrected according to Section 3 . The diffusion is slowed down by CB but there is definitively no indication of strong localization.

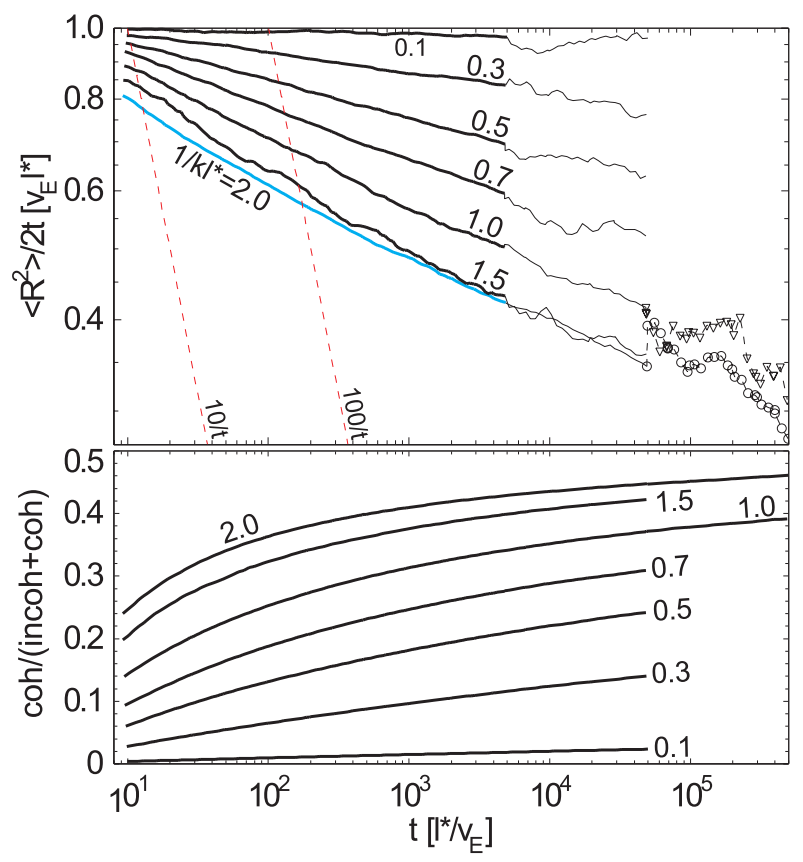

Fig. 7. Simulations according to a corrected self-attracting random walk in $2 \mathrm{D}$.

value $^{5}$, whereas in $2 \mathrm{D}$ the diffusion follows another power law, at least up to $5 \times 10^{5}$ scattering events.

\footnotetext{
5 Again, we want to emphasize that we do not say that there is no localization in $1 \mathrm{D}$. However, it is not sufficient solely to consider the ladder and the most crossed diagrams.
}

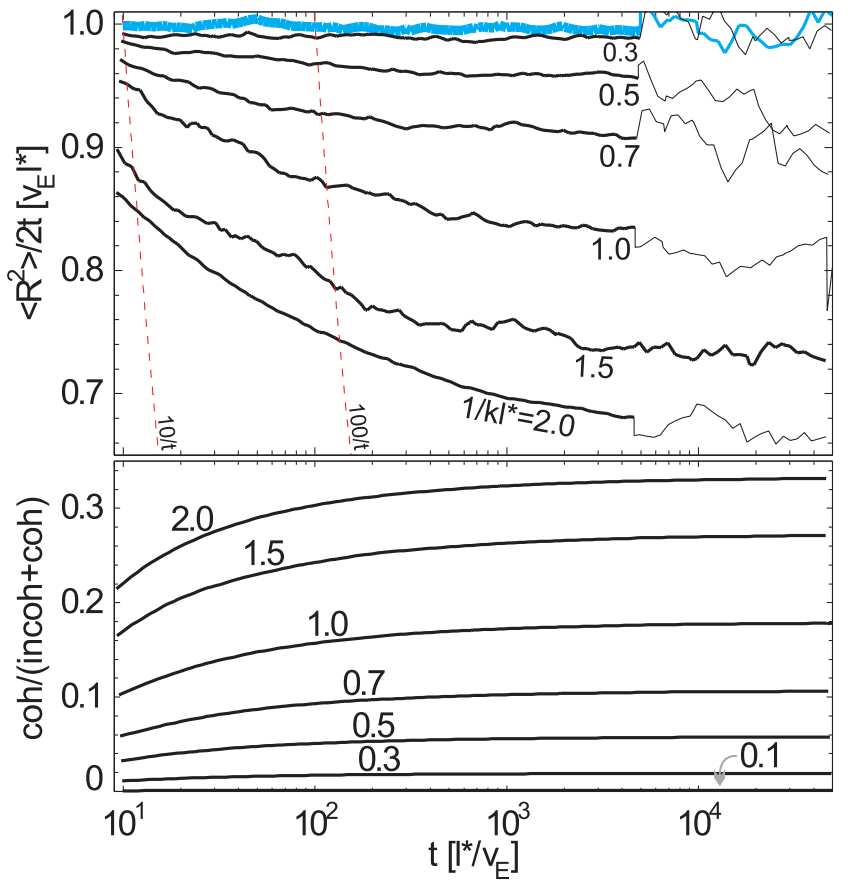

Fig. 8. Simulations according to a corrected self-attracting random walk in $3 \mathrm{D}$. Note that here the $\mathrm{CB}$ return probability does not even reach the maximum value of 0.5 .

\section{Summary}

We have studied the influence of coherent backscattering on wave propagation in disordered media in a selfattracting random walk model. Despite its simplicity, this model - before the correction with respect to overlapping CB-regions - well reflects the findings of other theories and gives a very intuitive picture of the underlying physics. However, using this model, it was possible for the first time to account for corrections on the length scale of $k_{\mathrm{e}}^{-1}$ necessary in the strongly scattering regime. Within this model, these corrections eliminate all indicators of strong localization in the context with $\mathrm{CB}$.

It would be interesting to study whether this model can be mapped onto more rigorous theories and whether refinements as suggested here would also lead to the disappearance of strong localization there.

We thank R. Klein and H. Stark for fruitful discussions. Financial support from the Optics Center Konstanz is acknowledged.

\section{References}

1. M. Rusek, A. Orlowski, Localisation of light in threedimensional disordered dielectrics, edited by V.A. Markel, T.F. George (Wiley, New York, 201, 2001).

2. For the introductory part we refer to the books of P. Sheng and references there: P. Sheng, Scattering and Localization of Classical Waves in Random Media (World Scientific, Singapore, 1990).

3. P. Sheng, Introduction to Wave Scattering, Localization and Mesoscopic Phenomena (Academic, San Diego, 1995). 
4. P.W. Anderson, Phys. Rev. 109, 1492 (1959) .

5. P.W. Anderson, Phil. Mag. B 52, 505 (1985) .

6. Here, we refer to the following book article and references there: R. Lenke, G. Maret, Scattering in Polymeric and Colloidal Systems, edited by W. Brown, K. Mortensen (Gordon and Breach, Scientific, 2000), pp. 1-73.

7. G. Bergmann, Phys. Rep. 107, 1-58 (1984).

8. A.A. Golubentsev, Sov. Phys. JETP 59, 26 (1984).

9. F.C. MacKintosh, S. John, Phys. Rev. B 37, 1884 (1988).

10. R. Lenke, G. Maret, Eur. Phys. J. B 17, 171 (2000).

11. R. Lenke, R. Lehner, G. Maret, Europhys. Lett. 52, 620 (2000).

12. G. Labeyrie, F. de Tomasi, J.-C. Bernard, C.A. Müller, C. Miniatura, R. Kaiser, Phys. Rev. Lett. 83, 5266 (1999).

13. D.S. Wiersma, P. Bartolini, A. Lagendijk, R. Righini, Nature 390, 671 (1997).
14. F.J.P. Schuurmans, M. Megens, D. Vanmaekelbergh, A. La-gendijk, Phys. Rev. Lett. 83, 2183 (1999).

15. F. Scheffold, R. Lenke, R. Tweer, G. Maret, Nature 398, 206 (1999), comment in [13].

16. This model was presented at the workshop Propagation dans les Milieux Hétérogènes: Diffusion et Conditions aux Limites, 10-11.5.2001, Paris for the first. The discussion principally showed that it can be accepted - at leat qualitatively - as an equivalent representation of current theories describing the influence of $\mathrm{CB}$ on multiple scattering.

17. This behavior is known for a self-avoiding random walk, where $b>0$ : M. Doi, S.F. Edwards, The Theory of Polymer Dynamics (Clarendon Press, Oxford 1989).

18. N.G. van Kampen, Stochastic processes in physics and chemistry (Elsevier, Amsterdam, 1997). 